\title{
Risk of Infectious Disease Outbreaks by Imported Cases with Application to the European Football Championship 2012
}

\author{
Attila Dénes, ${ }^{1}$ Péter Kevei, ${ }^{2}$ Hiroshi Nishiura, ${ }^{3,4}$ and Gergely Röst ${ }^{1}$ \\ ${ }^{1}$ Bolyai Institute, University of Szeged, Aradi vértanúk tere 1, Szeged 6720, Hungary \\ ${ }^{2}$ MTA-SZTE Analysis and Stochastics Research Group, Bolyai Institute, Aradi vértanúk tere 1, Szeged 6720, Hungary \\ ${ }^{3}$ School of Public Health, The University of Hong Kong, 100 Cyberport Road, Hong Kong \\ ${ }^{4}$ PRESTO, Japan Science and Technology Agency, Saitama, Japan
}

Correspondence should be addressed to Attila Dénes; denesa@math.u-szeged.hu

Received 28 September 2012; Accepted 27 November 2012

Academic Editor: Charles J. Mode

Copyright (C) 2013 Attila Dénes et al. This is an open access article distributed under the Creative Commons Attribution License, which permits unrestricted use, distribution, and reproduction in any medium, provided the original work is properly cited.

\begin{abstract}
The European Centre for Disease Prevention and Control called the attention in March 2012 to the risk of measles in Ukraine among visitors to the 2012 UEFA European Football Championship. Large populations of supporters travelled to various locations in Poland and Ukraine, depending on the schedule of Euro 2012 and the outcome of the games, possibly carrying the disease from one location to another. In the present study, we propose a novel two-phase multitype branching process model with immigration to describe the risk of a major epidemic in connection with large-scale sports-related mass gathering events. By analytic means, we calculate the expected number and the variance of imported cases and the probability of a major epidemic caused by the imported cases in their home country. Applying our model to the case study of Euro 2012 we demonstrate that the results of the football games can be highly influential to the risk of measles outbreaks in the home countries of supporters. To prevent imported epidemics, it should be emphasized that vaccinating travellers would most efficiently reduce the risk of epidemic, while requiring the minimum doses of vaccines as compared to other vaccination strategies. Our theoretical framework can be applied to other future sport tournaments too.
\end{abstract}

\section{Introduction}

The European Centre for Disease Prevention and Control reported a measles outbreak in Ukraine with more than 11,000 cases from the beginning of 2012 until the end of June 2012 $[1,2]$. The 2012 UEFA European Championship (Euro 2012) took place in Ukraine and Poland between 8 June and 1 July 2012, attracting several hundreds of thousands of football fans to these countries [3]. Susceptible visitors not only had a high risk of being infected, but also geographically propagating the epidemic to other countries.

We introduce a discrete time Markov chain model, which is an adaptation of a multitype Galton-Watson process with immigration to give a mathematical model for the evolution of the epidemic. Thus, we calculate the risk of epidemics connected to sports-related mass gathering events. Our model consists of two parts, the first one describing the spread of the disease during the championship in the host country, while the second part models the spread of the disease by fans returning to their home countries.

We apply our model to the special case of measles epidemics in Ukraine during the Euro 2012. Four of the eight host cities of this championship are in Ukraine (Kiev, Kharkiv, Lviv and Donetsk); one of these, Lviv, is situated in the western region where the prevalence is the highest and vaccination coverage remained the lowest in the country. Games of the group phase took place in the four Ukrainian cities for groups B and D including Denmark, Germany, Netherlands, Portugal and Ukraine, England, France, Sweden [4]. Two of the quarterfinals, one of the semifinals as well as the final took place in Ukraine, so Spain and Italy also played some games in Ukraine. The suboptimal measles vaccination coverage in many European countries poses a risk of measles epidemics caused by fans returning from Euro 2012. Here we study the impact of different outcomes of Euro 2012 on the probability of post-tournament measles epidemics in 
the participating countries and compare the effectiveness of different vaccination strategies by target host in reducing the risk of imported epidemics in other countries after Euro 2012. We discuss the applicability of our approach to other future events as well.

The rest of the paper is organized as follows. In Section 2 we describe the general mathematical model. In Section 3 we compute the probability of major epidemic in France after Euro 2012, while in Section 4 we compare the results with Euro 2008. Finally, we close with a discussion on the applicability of our model for other sports-related mass gathering events. In the appendix we calculate explicitly the expected number and the variance of infectious cases imported to the home country by supporters.

\section{Methods}

Since the supporter group spends a relatively short time in the infected area, it is possible that nobody gets infected, in which case there is no increased chance for epidemic in the home country. It is also clear that the risk of a huge epidemic is larger when five infected individuals arrive home (maybe to different parts of the country) than in the case when only one infectious supporter arrives. The fact that the number of infected supporters is zero, one, or five is just a matter of chance; thus, a deterministic model does not serve for our purposes in this case. It is well known (see [5]) that early stages of an epidemic in a large population can be approximated by branching processes, where having a descendant means infecting somebody. This exactly fits to our model, because in the host country the supporters spend short time (up to a month, say), and after returning to the home country we are only interested in the probability of a major epidemic, that is, in the early stage of a possible epidemic. To determine the final number of infectious individuals and/or the duration of the epidemic a mixture of a stochastic and a deterministic model is more appropriate. For general use of stochastic epidemic models we refer to a recent survey by Britton [5].

To describe the importation dynamics in the simplest manner, as a mathematical model, we propose a branching process with immigration. For simplicity, consider a single supporter population $S$ from a country $F$ which follows the matches of the team during the tournament, and ignore the interaction with other supporter groups. They can contract the disease from the local population or from each other. We define a discrete time Markov chain model, which is an adaptation of a multitype Galton-Watson process with immigration. We say that an individual is of type- $j$ if he/she contracted the infection exactly $j$ days ago. The model is divided into two phases: the first phase takes $T$ days and corresponds to the time spent in the host country, while the second phase describes the process upon returning to the home country. Let $m$ be the mean latent period and $k$ the mean infectious period of the disease (in days); that is, a newly infected individual becomes infectious only after $m$ days, and remains infectious for additional $k$ days. We assume these as constants. Denote by $\mathbf{X}_{t}$ the integer vector of infected individuals in population $S$ on day $t \leq T$ where $X_{t}(j)$, the number of type- $j$ individuals, is the number of infected individuals in population $S$ who got infected $j$ days ago, $j=$ $1,2, \ldots, m+k-1$. The evolution is the following. On day $t+1$ the newly infected individuals, that is, type- 1 individuals, can originate from the local population (immigrants), or from an $S$-individual who is infectious on day $t+1$ (which means that he/she got infected at least $m+1$ and at most $m+k$ days ago) and thus is of type $\geq m$ on day $t$ (offsprings). We assume that the force of infection from the local population to $S$ is constant during the first phase, and that the daily incidence produced by an infectious member of $S$ is also constant. Finally, for $j>1$, type- $j$ individuals arise only by getting one day older. After Phase 1 , the infected vector $\mathbf{X}_{T}$ returns to the home country, and each infected individual independently starts a simple single type Galton-Watson process.

In the following we describe the exact mathematical model.

2.1. Phase 1. Let $\mathbf{X}_{t}=\left(X_{t}(1), \ldots, X_{t}(d)\right)$ be a multitype Galton-Watson process with immigration, defined by

$$
\begin{gathered}
\mathbf{X}_{t}=\sum_{k=1}^{X_{t-1}(1)} \boldsymbol{\xi}_{t, k, 1}+\cdots+\sum_{k=1}^{X_{t-1}(d)} \boldsymbol{\xi}_{t, k, d}+\boldsymbol{\varepsilon}_{t}, \quad t \in\{1,2, \ldots, T\} \\
\mathbf{X}_{0}=\mathbf{0},
\end{gathered}
$$

where $\left\{\boldsymbol{\xi}_{t, k, i}, \boldsymbol{\varepsilon}_{t}: t \in\{1,2, \ldots, T\}, k \in \mathbb{N}, i \in\{1,2, \ldots, d\}\right\}$ are independent random vectors with nonnegative integer coordinates, such that $\left\{\boldsymbol{\xi}_{t, k, i}: t \in\{1,2, \ldots, T\}, k \in \mathbb{N}\right\}$ are identically distributed and $\left\{\boldsymbol{\varepsilon}, \boldsymbol{\varepsilon}_{t}: t \in\{1,2, \ldots, T\}\right\}$ are also identically distributed. Here the offsprings $\xi$ 's correspond to the new infections originated from an $S$-individual, while the immigrants $\boldsymbol{\varepsilon}$ 's correspond to new infections originated from the local population. Introduce the generating functions:

$$
\begin{gathered}
F_{t}(\mathbf{z})=\mathbf{E z}^{\mathbf{X}_{t}}, \quad G_{i}(\mathbf{z})=\mathbf{E} \mathbf{z}^{\xi_{1,1, i},} \\
\mathbf{G}(\mathbf{z})=\left(G_{1}(\mathbf{z}), \ldots, G_{d}(\mathbf{z})\right), \quad H(\mathbf{z})=\mathbf{E z}^{\boldsymbol{\varepsilon}},
\end{gathered}
$$

where $\mathbf{x}^{\mathbf{k}}=x_{1}^{k_{1}} \cdots x_{d}^{k_{d}}$. In the following boldface symbols $\mathbf{x}, \mathbf{y}, \mathbf{z}$ stand for $d$-dimensional vectors.

It is easy to show that the recursion $F_{t}(\mathbf{z})=$ $F_{t-1}(\mathbf{G}(\mathbf{z})) H(\mathbf{z})$ holds. Let $\mathbf{G}^{k}$ denote the $k$-fold iteration of $\mathbf{G}$, that is, $\mathbf{G}^{0}(\mathbf{z})=\mathbf{z}$ and $\mathbf{G}^{t+1}=\mathbf{G}^{t} \circ \mathbf{G}$. Then an induction argument shows (see Quine [6]) that the generating function of the $t$ th generation is

$$
F_{t}(\mathbf{z})=\prod_{k=0}^{t-1} H\left(\mathbf{G}^{k}(\mathbf{z})\right) .
$$

Up to now we did not use any particular property of the branching structure. However, note that in our case we have the following. The immigrants are always of type-1; thus, the generating function is in fact a one-variable function, that is, $H(\mathbf{z})=H\left(z_{1}, \ldots, z_{d}\right)=H\left(z_{1}\right)$. We also have $d=$ $m+k-1$. For $j=1,2, \ldots, m-1$ every type- $j$ particle has 
exactly one descendant of type- $(j+1)$ (the individual already infected is still not infectious, only getting one day older), thus $G_{j}(\mathbf{z})=z_{j+1}$, while for $j=m, m+1, \ldots, m+k-1$ the type$j$ individuals are already infecting and also getting one day older, so $G_{j}(\mathbf{z})=z_{j+1} G_{S}\left(z_{1}\right)$, with $G_{S}(z)$ being the generating function of the infected individuals on one day by a single infectious individual in $S$. Without the vector notation we have

$$
\begin{gathered}
X_{t}(1)=\sum_{i=m}^{m+k-1} \sum_{j=1}^{X_{t-1}(i)} \xi_{t, i, j}+\varepsilon_{t}, \\
X_{t}(j)=X_{t-1}(j-1), \quad j=2, \ldots, m+k-1,
\end{gathered}
$$

where $\left\{\xi_{i, j, t}: i=m, \ldots, m+k-1, j \in \mathbb{N}, t=1,2, \ldots, T\right\}$ are iid random variables with generating function $G_{S}$.

2.2. Phase 2. Phase 2 starts with the infected vector $\mathbf{X}_{T}$ arriving home. In this stage there is no immigration, and since the infected individuals stay home there is no point on registering the different types; hence, instead of counting the days we count the generation: $Y_{0}$ is the number of individuals who are infected by $\mathbf{X}_{T}, Y_{1}$ is the number of individuals who are infected by $Y_{0}$, and so forth. That is, the process now can be described by a single type Galton-Watson process. However, the first step is different, because the different types have different meanings. Individuals of type- $j, j \leq m$, spend all their infectious days in the home country, while individuals of type- $(m+j), j=1,2, \ldots, k-1$, spend only $k-j$ infectious days in the home country. Let $G_{F}(z)$ denote the generating function of the infected individuals on one day by a single infectious individual in the home country. Let $Y_{0}$ be the number of individuals who were infected by $\mathbf{X}_{T}$, then we have

$$
Y_{0}=\sum_{i=1}^{m} \sum_{j=1}^{X_{T}(i)} \xi_{i, j}+\sum_{i=m+1}^{m+k-1} \sum_{j=1}^{X_{T}(i)} \xi_{i, j},
$$

where $\left\{\xi_{i, j}: i=1,2, \ldots, m+k-1, j \in \mathbb{N}\right\}$ are independent random variables, and $\left\{\xi_{i, j}: i=1,2, \ldots, m, j \in \mathbb{N}\right\}$ are iid with generating function $G_{F}^{k}$, and for $i \in\{m+1, \ldots, m+k-1\}$, $\left\{\xi_{i, j}: j \in \mathbb{N}\right\}$ are iid with generating function $G_{F}^{m+k-i}$. Using the representation above for the generating function of $Y_{0}$ we obtain

$$
\begin{aligned}
h(z) & :=\mathbf{E} z^{Y_{0}} \\
& =F_{T}\left(G_{F}^{k}(z), \ldots, G_{F}^{k}(z), G_{F}^{k-1}(z), \ldots, G_{F}(z)\right) .
\end{aligned}
$$

Now, all who were infected after this step spend their infectious days in the home country, so the process now is a simple single type Galton-Watson process with offspring generating function $g(z)=G_{F}^{k}(z)$, starting from random initial state $Y_{0}$.

If this simple Galton-Watson process is critical or subcritical, that is, $g^{\prime}(1) \leq 1$, then the process dies out almost surely, regardless of the distribution of $Y_{0}$; that is, there is no major epidemic in this case. In the supercritical case, when $g^{\prime}(1)>$ 1 , the probability that starting from a single individual the process dies out is the unique root in $(0,1)$ of the equation $g(x)=x$. Let $q$ denote this extinction probability. The process starting from $Y_{0}$ dies out if all the $Y_{0}$ branches die out, which has probability $q^{Y_{0}}$. That is, the probability of extinction of the whole process is

$$
\mathbf{P}\{\text { extinction }\}=\mathbf{E} q^{Y_{0}}=h(q),
$$

with $h$ as in (6).

\section{Computations for the European Football Championship 2012}

3.1. Risk of Measles Outbreak Depends on the Results of the Football Games. In this section we apply the results to the measles epidemic in Ukraine during the 2012 UEFA European Football Championship. For illustratory purposes, we have chosen France as a prototype for describing the results. In fact, as being amongst the four favourites for the European championship title [7], France was likely to be amongst the teams with the most supporters, while having low vaccination coverage against measles, posing an elevated risk of imported epidemic caused by supporter cases after Euro 2012. We compare the following three scenarios, one of which is the real situation in Euro 2012, while the two others are hypothetical cases representing the extremes for France by means of total time spent in Ukraine (see also Figure 1):

(a) France is eliminated in the group stage, thus playing only three games in Ukraine between June 11 and June 19 (hypothetical case);

(b) France finishes second in the group and is eliminated in the quarterfinals, playing four games in Ukraine between June 11 and June 23 (this is what actually happened);

(c) France finishes second in the group, and gets into the final, thus playing six games between June 11 and July 1, all in Ukraine (hypothetical case).

We assume that the supporter population is staying in Ukraine as long as the team continues to play games. The total length of stay would be the length of games plus one extra day due to international travel, and thus in the three cases we have $T=10(\mathrm{a}), T=14(\mathrm{~b})$, and $T=21$ (c).

For our computations we set $m=9, k=9$ [8]. Since measles is generally rare in Europe, the effective reproduction number in France $R_{F}=g^{\prime}(1)$ is determined by the basic reproduction number $R_{0}$ of measles and the effective vaccination coverage $v_{F}$ in France (e.g., the fraction of population that is immunized and protected) due to $R_{F}=R_{0}\left(1-v_{F}\right)$. The basic reproduction number $R_{0}$ is estimated between 12 and 18. For computations we use $R_{0}=15$. Thus, $R_{F}$ is realistically assumed to be in the range 1-3 [9-12]. The contact pattern within the supporter group might be different from the general population, but still the effective reproduction number in Ukraine $R_{S}=k \beta=k G_{S}^{\prime}(1)$ is expected to be of the same magnitude as $R_{F}$ providing a reasonable range for $\beta$. Parameter $\lambda=H^{\prime}(1)$, which represents the expected number of daily infected individuals infected by members of the local 


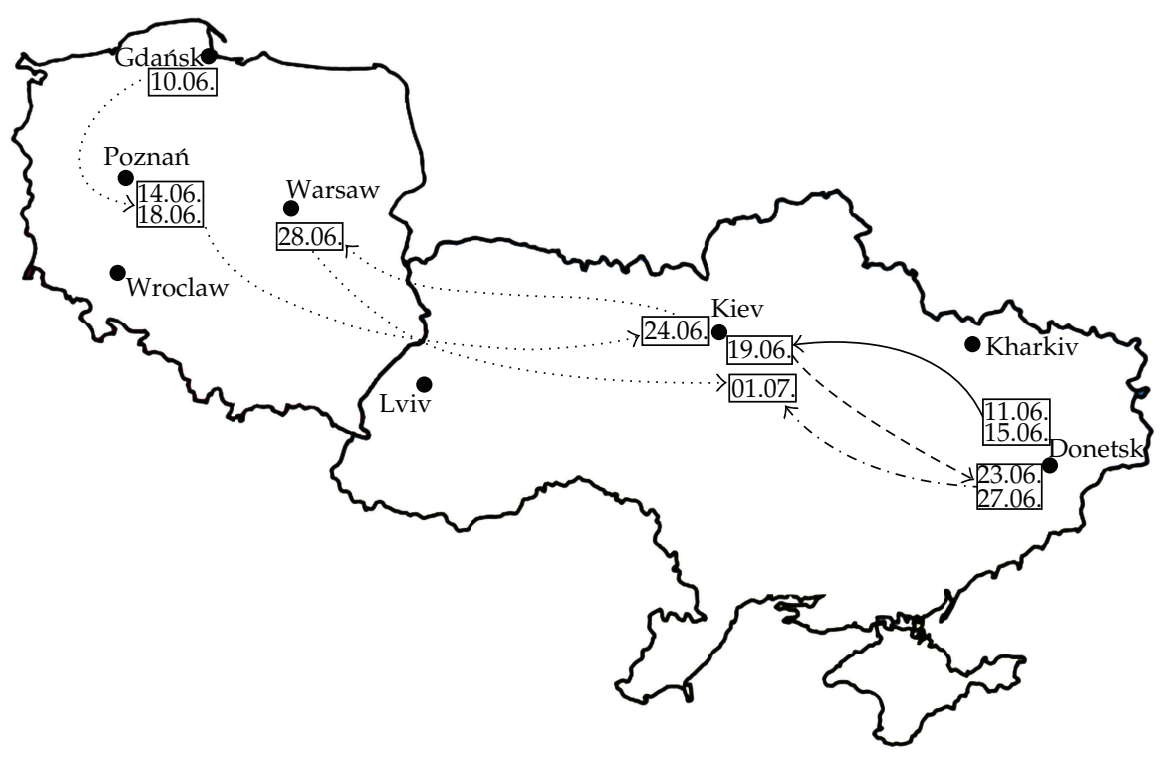

FIGURE 1: The movement of France during Euro 2012 and the dates of games. The solid arrow corresponds to the group stage, the dashed arrow corresponds to additional games in scenarios (b) and (c), and the dot-dashed arrow corresponds to the hypothetical case of getting into the final (scenario (c)). The dotted arrows represent the movement of Italy (chosen randomly for illustratory purposes) during the tournament.

population, is the most difficult to estimate, as this is given by a combination of several factors: the morbidity of measles in Ukraine during the tournament, the contact patterns between and within local and supporter populations, the size of the supporter group, and the level of susceptibility in this group. We scanned a large domain $[0,0.125]$ for $\lambda$. A person having measles changes his mixing and contact patterns due to the infection, but it should be noted that generally such a change in social behaviour is accounted for the estimate of $R_{0}$. Here we assume that individuals in the host country, home country, and visitor populations modify their social behaviour similarly after contracting the disease; thus, our three key parameters $\lambda, \beta$, and $R_{F}$ are all proportional to $R_{0}$.

By the nature of the immigration and the offspring distributions it is natural to assume that these are Poisson, or compound Poisson distributed. We calculate the extinction probabilities in two cases: when the offspring and immigration distributions are Poisson distributions and when they are negative binomial distributions. In the appendix we explicitly calculate some relevant quantities. We assume that the expectations of the total number of daily new infections from the local population $(\lambda)$, the expectation of daily new infections by one infectious individual from the supporter population $(\beta)$, and the expectation of daily new infections by one infectious individual in the home country $(\gamma)$ are known and choose the parameters of the generating functions accordingly. Note that $R_{F}=9 \gamma$.

Assuming that both the immigration and the offspring distributions are Poisson, we have

$$
\begin{array}{ll}
H(z)=e^{\lambda(z-1)}, & G_{S}(z)=e^{\beta(z-1)}, \\
G_{F}(z)=e^{\gamma(z-1)}, & g(z)=e^{9 \gamma(z-1)} .
\end{array}
$$

A random variable $X$ has negative binomial distribution with parameters $r>0$ and $p \in(0,1)$, if $\mathbf{P}\{X=k\}=$ $\left(\begin{array}{c}k+r-1 \\ r-1\end{array}\right)(1-p)^{r} p^{k}, k=0,1,2, \ldots$, where the binomial coefficient is defined by $\left(\begin{array}{c}k+r-1 \\ r-1\end{array}\right)=(k+r-1)(k+r-2) \cdots r / k$ !. The generating function is

$$
\mathbf{E} z^{X}=\left(\frac{1-p}{1-p z}\right)^{r},
$$

so the expectation is $\mathbf{E} X=r p /(1-p)$. In the special case, when $r=1$ we obtain the geometric distribution. Assuming geometric distribution for the immigration and the one-day infections

$$
\begin{gathered}
H(z)=[1+\lambda-\lambda z]^{-1}, \quad G_{S}(z)=[1+\beta-\beta z]^{-1}, \\
G_{F}(z)=[1+\gamma-\gamma z]^{-1},
\end{gathered}
$$

(the parameters are chosen to make the corresponding expectations to be $\lambda, \beta$, and $\gamma$ resp.), and using that $G_{F}^{k}=g$ we necessarily have

$$
g(z)=[1+\gamma-\gamma z]^{-9}
$$

Figure 2 shows that the risk in scenario $T=21$ can be twice as large as in scenario $T=10$.

Comparing Figures 2(a) and 2(b) we see that there is no much difference in the behaviour of the extinction probabilities. In the Poissonian case the extinction probability is slightly larger than in the negative binomial case, corresponding to the same parameter values. Therefore, in the following we assume the Poissonian setup.

The extinction probabilities cannot be computed explicitly. This is because $q$, the probability of extinction starting 
from one individual, cannot be calculated explicitly neither in the Poissonian nor in the negative binomial case. We numerically solve the equation for different values of $\gamma$ running from 0.111 up to 0.334 ; that is, $R_{F}$ varies in the range $1-3$ (recall that the expected value of offsprings in Phase 2 is $9 \gamma$ ). Then we substitute these values into the explicitly known generating function $h$, given in (6).

3.2. Vaccination and the Risk of Epidemics after Euro 2012. We compare the effectiveness of three potential vaccination strategies in reducing the risk of imported major epidemic:

(i) vaccination of the general population in France;

(ii) vaccination of the general population in Ukraine;

(iii) vaccination of football-associated travellers between France and Euro 2012 venues.

To consider (i), note that increasing the vaccination rate $v_{F}$ decreases each of the parameters in our model. That is, $9 \gamma=R_{F}=R_{0}\left(1-v_{F}\right), \lambda=\lambda_{1}\left(1-v_{F}\right)$, and $\beta=\beta_{1}\left(1-v_{F}\right)$. We plotted the risk of major epidemic $p$ as a function of $v_{F}$ in Figure 3, and the expected number of imported cases in Figure 4.

Figure 3(a) shows a milder measles epidemics in Ukraine, and Figure 3(b) corresponds to a more severe situation. We may notice that it is particularly worthwhile to increase the immunization rate in France if the epidemic is severe in Ukraine, because in this case we could observe a steep decline in the risk as $v_{F}$ increased beyond 0.84 (which is, roughly speaking, consistent with the reported present coverage in the country). Increasing $v_{F}$ has the benefit of decreasing the risk of outbreaks by imported cases unrelated to Euro 2012.

On the other hand, elevating the vaccination level $v_{U}$ of the local Ukrainian population decreases $\lambda=\lambda_{2}\left(1-v_{U}\right)$. The current value of $v_{U}$ is reported to be about 0.5 [13]. Given the difference between the total populations of Ukraine and France, increasing $v_{F}$ by one unit requires the same amount of vaccines as increasing $v_{U}$ by 1.4 units. However, the computations show that $p$ is much less sensitive to $v_{U}$ than to $v_{F}$ (Figure 5), because small reduction in the risk of infection during travel may only slightly reduce the imported cases.

Targeted vaccination of football visitors reduces both $\lambda=$ $\lambda_{1}\left(1-v_{T}\right)$ and $\beta=\beta_{1}\left(1-v_{T}\right)$ where $v_{T}$ is the level of immunization in $S$ (i.e., in the absence of targeted vaccination of travellers, it is assumed that $v_{T}=v_{F}$ ). Figure 6 shows the efficiency of this strategy in the case of a milder and a more severe Ukrainian measles epidemic. If the vaccination history was perfectly known, elevating from 0.84 to 0.94 would be achieved by vaccinating $10 \%$ of the travellers from France (targeting the unvaccinated ones). Such an intervention can halve the risk with relatively small efforts. It should be noted that elevating the coverage $v_{T}$ would require the smallest number of doses (as compared to conducting mass vaccinations in other scenarios) as vaccinating the supporters requires only a couple of thousands of doses.

\section{Comparison with Euro 2008}

In contrast to Euro 2012, here we descriptively review the measles outbreaks which are likely associated with Euro 2008 and other mass gathering events. The 2008 UEFA European Football Championship (Euro 2008) took place in Austria and Switzerland from 7 to 29 June 2008. Significant measles outbreaks were reported in both of the host countries before the championship [14]. However, that situation was different from this year's in several aspects. First of all, the vaccination coverage is much higher in Switzerland and Austria than in Ukraine, and consequently, as the morbidity data show, the measles outbreak in 2012 in Ukraine is of significantly larger scale than the one in the two host countries four years ago [15]. It is also likely that Euro 2008 did not elevate the relative number of travellers as much as Euro 2012 in Ukraine as the two host countries of Euro 2008 are close to several of the participating countries and most host cities are popular tourist destinations, hosting a large number of visitors even without the football championship. As it has been pointed out in [16], a large scale mass gathering can even discourage regular tourists to visit the given cities to avoid the crowdedness, as happened in 2008 during the Olympic Games in Beijing. The media reported a similar phenomenon in London during the 2012 Olympic Games. In other cases (e.g., Sydney 2000), there was a surge of travellers, and we can assume the same for Ukraine as well.

For Euro 2008 we chose Germany as the German national team reached the final of the championship, which means that their supporters spent 21 days in Austria and Switzerland, and WHO reports a suboptimal coverage of $83-89 \%$ for the second dose of measles-containing vaccine in Germany [17]. Taking into account the number of measles cases in Austria/Switzerland in 2008 and in Ukraine in 2012, and the population of these countries, we can expect the parameter $\lambda$ to be approximately ten times smaller for Euro 2008 than for Euro 2012. Assuming Poisson distribution, calculating with $\lambda=0.004$ and $\beta=0.27$, formula (A.4) says that the probability of no imported infection is 0.92 ; that is the probability of major epidemic is less than 0.08 , which is significantly smaller than the probabilities for Euro 2012.

Data from 2008 show that in several participating countries (e.g., France, Germany, Spain, and Switzerland) there were increases in the number of measles cases after Euro 2008 compared to the same period of the year in 2007 [1821]. However, based on available data, a direct link cannot be established between Euro 2008 and these outbreaks.

\section{Other Sports-Related Mass Gatherings}

As pointed out in [22], the last two European football championships are not unique in the sense that curiously, the football championships seem to coincide with measles outbreaks. Apart from the two cases mentioned above, during the FIFA World Cup 2006 a large measles outbreak was ongoing in Germany (host country), while there was an outbreak in South Africa during the FIFA World Cup 2010. Furthermore, the Winter Olympic Games in 2010, held in Vancouver, were followed by a measles outbreak in British Columbia of about 


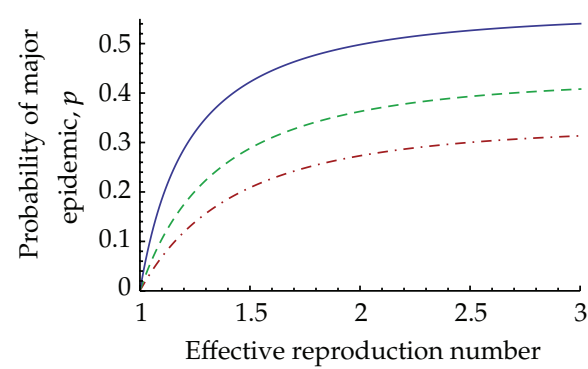

in France, $R_{F}$

(a)

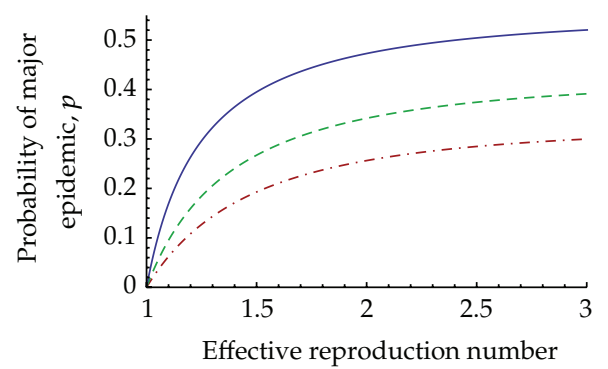

in France, $R_{F}$

(b)

Figure 2: The probability of a major epidemic as the function of the effective reproduction number in France in the Poissonian case (a) and in the negative binomial case (b). The parameters are $\lambda=0.04, \beta=0.27$. The solid curve is for $T=21$, the dashed is for $T=14$, and the dot-dashed is for $T=10$.

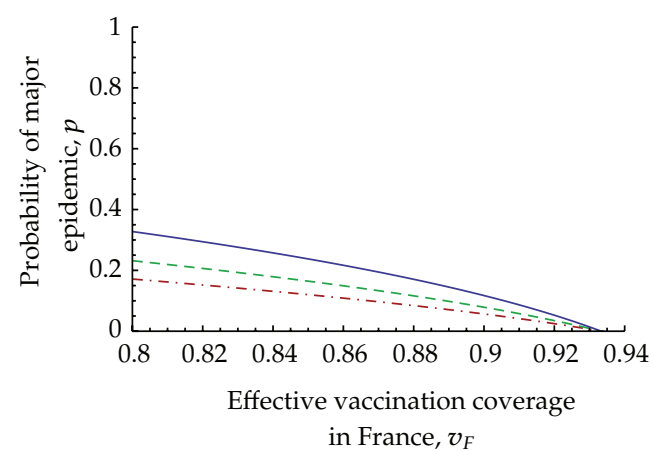

(a)

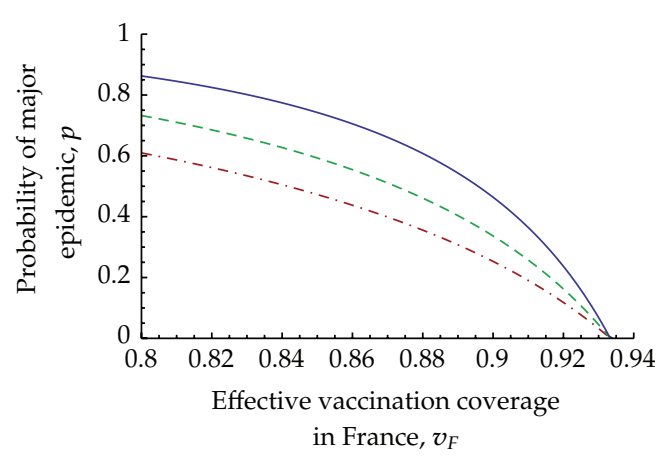

(b)

Figure 3: The probability of a major epidemic as the function of the immunization rate in France. The parameters are $\beta_{1}=1.8$, and $\lambda_{1}=0.1$ in (a) and $\lambda_{1}=0.5$ in (b). The solid curve is for $T=21$, the dashed is for $T=14$, and the dot-dashed is for $T=10$.

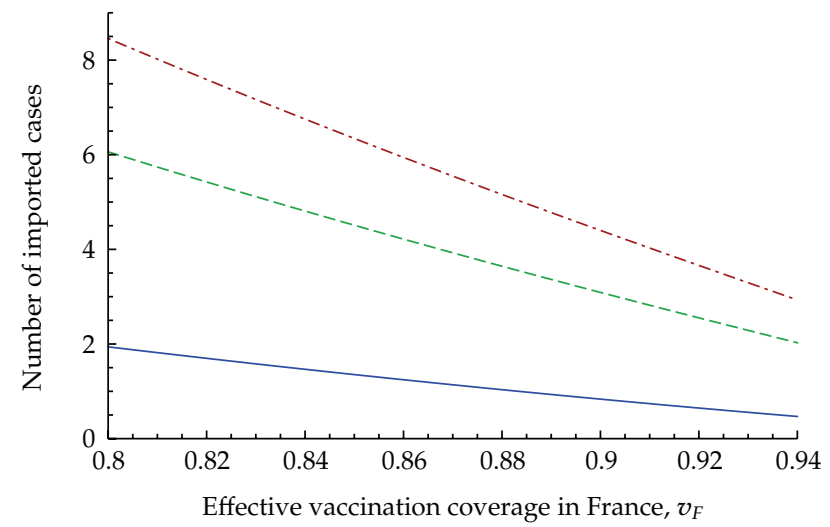

FIgURE 4: The solid curve is the expectation of the total number of imported cases in scenario (b) in the function of the immunization rate in France. At least with probability 0.75 the number of imported cases is smaller than the dashed curve and with probability 0.9 is smaller than the dot-dashed curve (calculated from Chebyshev's inequality). The parameter values are the same as in Figure 3(b).

80 cases following three separate importations, two of which were linked to the Olympic Games [23].

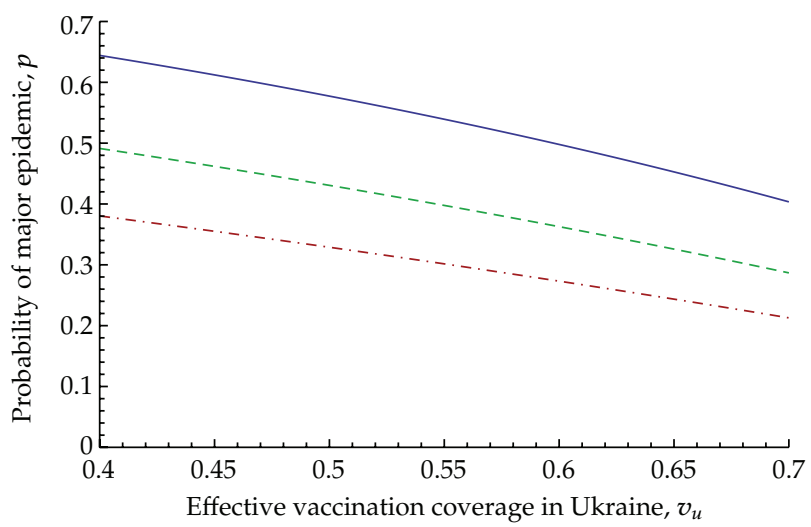

FIGURE 5: The probability of a major epidemic as the function of the immunization rate in Ukraine. The parameters are $R_{F}=2, \beta_{1}=1.8$, and $\lambda_{1}=0.1$. The solid curve is for $T=21$, the dashed is for $T=14$, and the dot-dashed is for $T=10$.

After Euro 2012, another sports related mass gathering event followed, the Summer Olympic Games in London. There were several alerts about measles in connection with the Olympic Games [24, 25]. However, there are several differences between football championships and the Olympic 


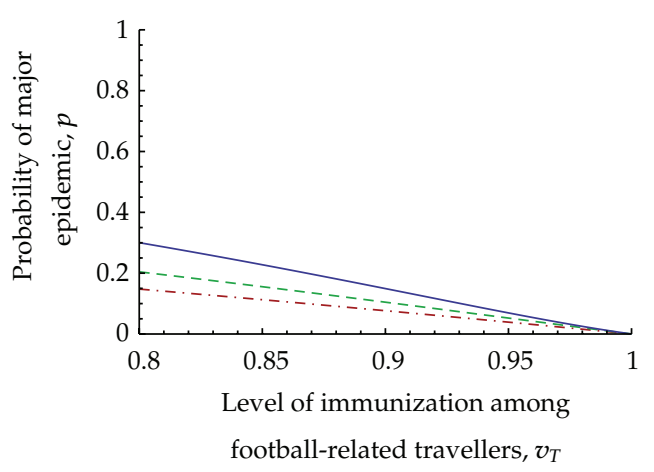

(a)

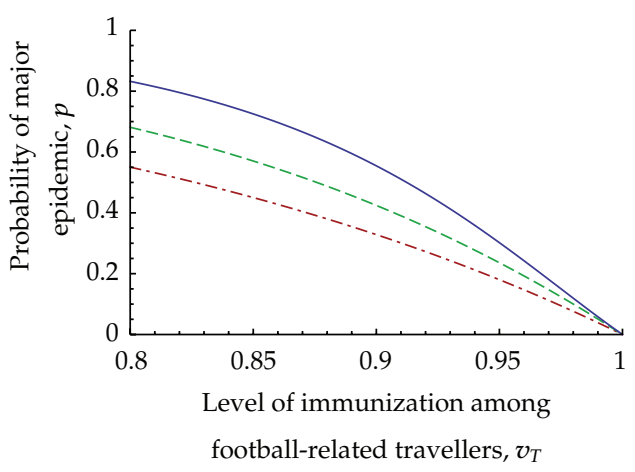

(b)

FIGURE 6: The probability of a major epidemic as the function of the immunization rate in the supporter group. The parameters are $R_{F}=2$, $\beta_{1}=1.8$, and $\lambda_{1}=0.1$ in (a) and $\lambda_{1}=0.5$ in (b). The solid curve is for $T=21$, the dashed is for $T=14$, and the dot-dashed is for $T=10$.

Games. Football championships have a special tournament structure and huge groups of fans moving together following their national teams, which is not typical for the Olympic Games. Football championships are hosted by several cities, while the Olympic Games are held (apart from some minor events) in one city. This means that our model fits rather for sport events which have the tournament structure like football World Cup and European Championship. With an efficient monitoring after Euro 2012, it may be possible to refine our parameters and prepare more realistic risk assessments using our approach for the forthcoming major championships such as FIFA World Cup 2014 in Brazil and Euro 2016 in France. The low vaccination rate and the recent and ongoing measles epidemics in France $[26,27]$ suggest that there will be a risk of measles during Euro 2016 as well.

\section{Discussion}

We constructed and applied a stochastic model to investigate the risk of imported epidemics caused by visitors returning from a sports related mass gathering event to their home countries after the tournament. For the sake of simplicity, we considered a single supporter population, while a realistic situation of course involves many additional complicating factors including movements within the host country and interactions between supporters and local population. We introduced a discrete time Markov chain model with two phases, which is an adaptation of a multitype Galton-Watson process with immigration as a mathematical model and derived several analytical relations for the expectations, variances and probabilities regarding key aspects of the process.

We applied our theoretical model to the measles epidemics in Ukraine during the 2012 UEFA European Football Championship, selecting the national team of France for illustratory purposes. Due to the uncertainties in social parameters, we considered a wide interval for the transmission rate between local and visitor populations. Our approach clearly demonstrated that the travel patterns depend on the schedule and the results of the football games, showing that the probability of a major measles epidemic in France could be greatly elevated by the successful outcomes of French games. Namely, the more successful the national team is in a football tournament, the higher the risk of a post-tournament imported measles epidemic would be in the home country. More importantly, we have compared different vaccination strategies and our study theoretically demonstrated that the risk of an imported measles epidemic by the visitors to Euro 2012 and other mass gatherings would be most efficiently reduced by vaccinating the visitors (travellers). Of course, vaccinating the entire French population would also be effective (which actually prevents the country from not only the risk from Euro 2012 but also any other epidemics to be imported), but in theory this option requires us to secure millions of doses. The optimal control by effectively targeting travellers is novel both in practical and theoretical sense, because the condensed interventions among travellers have been shown not to be very effective in preventing an epidemic (e.g., pandemic influenza) as long as there are arbitrarily large number of travellers. We have shown that it is worth focusing on travellers when the number is finite and in the manageable order. Unvaccinated travellers would likely be covered within a few thousand doses, and thus any country to respond to the associated risk is suggested to consider this option.

\section{Appendix}

We compute explicitly the expectation and variance of the overall number of infectious individuals arriving home after day $T$, that is, $Z:=\sum_{i=1}^{17} X_{T}(i)$, and also we compute the probability that there is no imported infection, that is, $\mathbf{P}\{Z=$ 0 \}. To do this we compute the generating function $F_{T}(\mathbf{z})$ given in (3) and then we use that

$$
\begin{gathered}
\mathbf{P}\{Z=0\}=F_{T}(\mathbf{0}), \quad \mathbf{E} Z=\sum_{i=1}^{17} \frac{\partial F_{T}}{\partial z_{i}}(\mathbf{1}), \\
\operatorname{Var} Z=\sum_{i, j}\left(\frac{\partial^{2} F_{T}}{\partial z_{i} \partial z_{j}}(\mathbf{1})-\mathbf{E} X_{T}(i) \mathbf{E} X_{T}(j)+\delta_{i, j} \mathbf{E} X_{T}(i)\right),
\end{gathered}
$$

with $\delta_{i, j}=1$ for $i=j$, and 0 otherwise. Note that one minus the probability of no imported infection is a trivial 
upper bound for the probability of a major epidemic, and it is independent of $\gamma$.

Assuming Poissonian offspring and immigration distribution, we have the following:

(i) $T=10$ : in this case everything is relatively easy to compute. For example, there is no imported infection if and only if each day the number of immigrants is 0 , which has probability $e^{-10 \lambda}$. We have

$$
\begin{gathered}
\mathbf{P}\{Z=0\}=e^{-10 \lambda}, \quad \mathbf{E} Z=\lambda(10+\beta), \\
\operatorname{Var} Z=\lambda\left(10+3 \beta+\beta^{2}\right) ;
\end{gathered}
$$

(ii) $T=14$ :

$$
\begin{gathered}
\mathbf{P}\{Z=0\}=e^{-14 \lambda}, \quad \mathbf{E} Z=\lambda(14+15 \beta), \\
\operatorname{Var} Z=\lambda\left(14+45 \beta+55 \beta^{2}\right) ;
\end{gathered}
$$

(iii) $T=21$ :

$$
\begin{gathered}
\mathbf{P}\{Z=0\}=e^{-17 \lambda-4\left(1-e^{-9 \beta}\right) \lambda}, \quad \mathbf{E} Z=\lambda\left(17+72 \beta+10 \beta^{2}\right), \\
\operatorname{Var} Z=\lambda\left(17+144 \beta+558 \beta^{2}+200 \beta^{3}+46 \beta^{4}\right) .
\end{gathered}
$$

In the negative binomial case for the different scenarios we have

(i) $T=10$ :

$$
\begin{aligned}
& \quad \mathbf{P}\{Z=0\}=(1+\lambda)^{-10}, \quad \mathbf{E} Z=\lambda(10+\beta), \\
& \operatorname{Var} Z=\lambda\left(10(1+\lambda)+\beta^{2}(2+\lambda)+\beta(3+2 \lambda)\right) ; \\
& \text { (ii) } T=14: \\
& \quad \mathbf{P}\{Z=0\}=(1+\lambda)^{-14}, \quad \mathbf{E} Z=\lambda(14+15 \beta), \\
& \operatorname{Var} Z=\lambda\left(14(1+\lambda)+15 \beta(3+2 \lambda)+\beta^{2}(70+55 \lambda)\right),
\end{aligned}
$$

(iii) $T=21$ :

$$
\begin{gathered}
\mathbf{P}\{Z=0\}=(1+\lambda)^{-17}\left(1+\lambda-\frac{\lambda}{(1+\beta)^{9}}\right)^{-4}, \\
\mathbf{E} Z=\lambda\left(17+72 \beta+10 \beta^{2}\right), \\
\operatorname{Var} Z=\lambda\left(17(1+\lambda)+72 \beta(2+\lambda)+6 \beta^{2}(105+88 \lambda)\right. \\
\left.+10 \beta^{3}(23+18 \lambda)+\beta^{4}(66+46 \lambda)\right) .
\end{gathered}
$$

Also note that in both cases the variance is large compared to the expectation, implying that the probability of no imported cases is large.

\section{Acknowledgments}

A. Dénes and G. Röst were supported by the European Research Council Starting Investigator Grant no. 259559, the Hungarian Scientific Research Fund OTKA K75517, and Bolyai Scholarship of the Hungarian Academy of Sciences. P. Kevei was supported by the TÁMOP-4.2.1/B-09/1/KONV2010-0005 Project and the Hungarian Scientific Research Fund OTKA PD106181. H. Nishiura received funding support from the JST PRESTO Program and The University of Hong Kong Seed Funding Program (Grant Code: 10208192).

\section{References}

[1] "Outbreak of measles in Ukraine and potential for spread in the EU, Rapid Risk Assessment," ECDC, 13 March 2012, http://ecdc.europa.eu/en/publications/Publications/20120314_ RA_Measles_Ukraine.pdf.

[2] "Communicable disease threats report," ECDC, Week 26, 24-30 June 2012, http://ecdc.europa.eu/en/publications/Publications/ CDTR_web_2012_6_28.pdf.

[3] B. R. Humphreys and S. Prokopowicz, "Assessing the impact of sports mega-events in transition economies: EURO 2012 in Poland and Ukraine," International Journal of Sport Management and Marketing, vol. 2, no. 5-6, pp. 496-509, 2007.

[4] "UEFA webpage," http://www.uefa.com/uefaeuro/season=2012 /tournament-calendar/index.html.

[5] T. Britton, "Stochastic epidemic models: a survey," Mathematical Biosciences, vol. 225, no. 1, pp. 24-35, 2010.

[6] M. P. Quine, "The multi-type Galton-Watson process with immigration," Journal of Applied Probability, vol. 7, no. 2, pp. 411422, 1970.

[7] "Bwin," http://www.bwin.com/Euro2012.

[8] R. D. Feigin, J. Cherry, G. J. Demmler-Harrison, and S. L. Kaplan, Feigin and Cherry's Textbook of Pediatric Infectious Diseases, Saunders, 6th edition, 2009.

[9] L'Institut de Veille Sanitaire (InVS), http://www.invs.sante.fr/ Dossiers-thematiques/Maladies-infectieuses/Maladies-a-prevention-vaccinale/Rougeole/Points-d-actualites.

[10] L. Fonteneau, J.-M. Urcun, C. Kerneur et al., "Couverture vaccinale des enfants gs de 11 ans scolariss en CM2, France, 2004-2005," Bulletin Épidémiologique Hebdomadaire, vol. 51-52, pp. 493-497, 2008 (French).

[11] I. P. du Châtelet, D. Antona, F. Freymuth et al., "Spotlight on measles 2010: update on the ongoing measles outbreak in france, 2008-2010," Euro Surveillance, vol. 15, no. 36, pp. 1-4, 2010 http://www.eurosurveillance.org/ViewArticle .aspx?ArticleId=19656.

[12] I. Bonmarin and D. Lvy-Bruhl, "Measles in France: the epidemiological impact of suboptimal immunisation coverage," Euro Surveillance, vol. 7, no. 4, p. 322, 2002, http://www.eurosurveillance.org/ViewArticle.aspx?ArticleId=322.

[13] "WHO epidemiological brief," no. 21, 2012, http://www. euro.who.int/__data/assets/pdf_file/0004/159475/WHO_EPI_ Brief_Feb_2012e.pdf.

[14] R. Strauss, P. Kreidl, M. Muscat et al., "The measles situation in Austria: a rapid risk assessment by an ECDC team and the outcome of an International Meeting in Vienna, Austria," Euro Surveillance, vol. 13, no. 17, 2008, http://www .eurosurveillance.org/ViewArticle.aspx?ArticleId=18852. 
[15] P. Kreidl, P. Buxbaum, F. Santos-O’Connor et al., "2008 European Football Championship-ECDC epidemic intelligence support," Euro Surveillance, vol. 13, no. 32, 2008, http://www .eurosurveillance.org/ViewArticle.aspx?ArticleId=18946.

[16] K. Khan, S. J. N. McNabb, Z. A. Memish et al., "Infectious disease surveillance and modelling across geographic frontiers and scientific specialties," The Lancet Infectious Diseases, vol. 12, no. 3, pp. 222-230, 2012.

[17] WHO, "Reported estimates of MCV coverage," http://apps.who .int/immunization_monitoring/en/globalsummary/timeseries/ tscoveragemcv.htm.

[18] I. Parent du Chtelet, D. Floret, D. Antona, and D. LvyBruhl, "Measles resurgence in France in 2008, a preliminary report," Euro Surveillance, vol. 14, no. 6, 2009, http://www.eurosurveillance.org/ViewArticle.aspx?ArticleId=19118.

[19] Robert Koch Institut SurvStatRKI, Abfrage der Meldedaten nach Infektionsschutzgesetz (IfSG) ber das Web, http://www3.rki.de/SurvStat/.

[20] "Resultados de la vigilancia epidemiolgica de las enfermedades transmisibles," Informe Anual, 2008, http://www .isciii.es/ISCIII/es/contenidos/fd-servicios-cientifico-tecnicos/ fd-vigilancias-alertas/fd-enfermedades/Informeanual2008.pdf.

[21] E. Delaporte, E. Jeannot, P. Sudre, C. A. W. Lazarevic, J. L. Richard, and P. Chastonay, "Measles in Geneva between 2003 and 2010: persistence of measles outbreaks despite high immunisation coverage," Euro Surveillance, vol. 16, no. 39, 2011, http://www.eurosurveillance.org/ViewArticle.aspx?ArticleId= 19980.

[22] Editorial team, "Spotlight on measles 2010," Euro Surveill, vol. 15, no. 17, 2010, http://www.eurosurveillance.org/ViewArticle.aspx?ArticleId=19559.

[23] "BC Centre for Disease Control," http://www.bccdc.ca/ resourcematerials/newsandalerts/healthalerts/Before+travelling+ensure+your+measles+vaccination+is+up+to+date.htm.

[24] "Surveillance report," European Monthly Measles Monitoring (EMMO) Issue 10, 2012, http://ecdc.europa.eu/en/publications/ Publications/1205-SUR-Measles-monthly-monitoring.pdf.

[25] "Centers for Disease Control and Prevention," http://wwwnc .cdc.gov/travel/notices/outbreak-notice/measles.htm.

[26] C. Huoi, J. S. Casalegno, T. Bnet et al., "A report on the large measles outbreak in Lyon, France, 2010 to 2011," Euro Surveillance, vol. 17, no. 36, 2012 http://www.eurosurveillance.org/ViewArticle.aspx?ArticleId=20264.

[27] "Measles and rubella monitoring," Surveillance report, ECDC, 2012, http://www.ecdc.europa.eu/en/publications/Publications/2012Sept_SUR_measles-rubella-monitoring.pdf. 


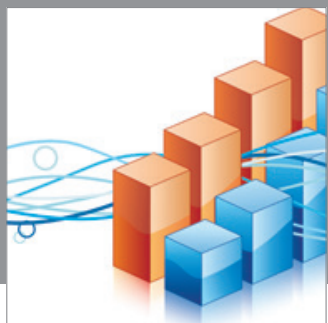

Advances in

Operations Research

mansans

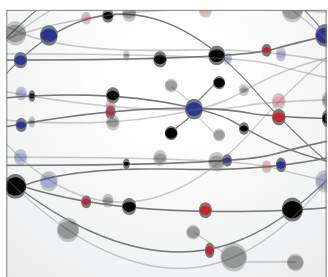

The Scientific World Journal
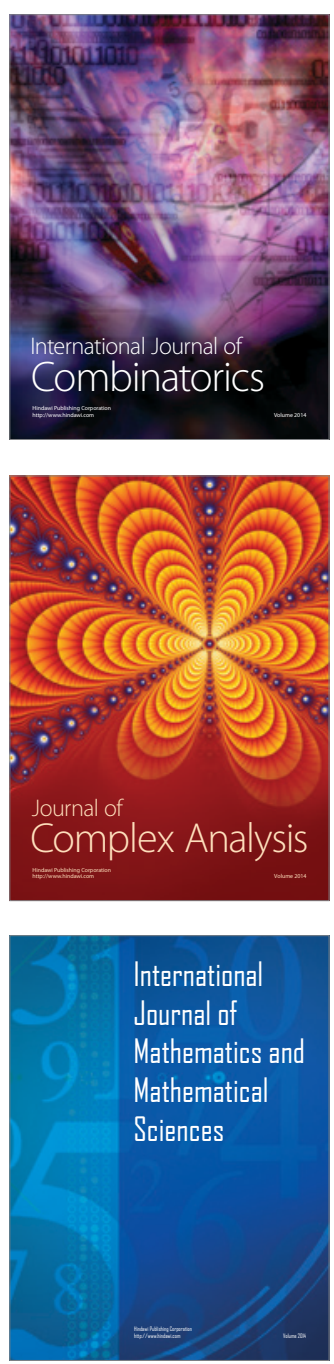
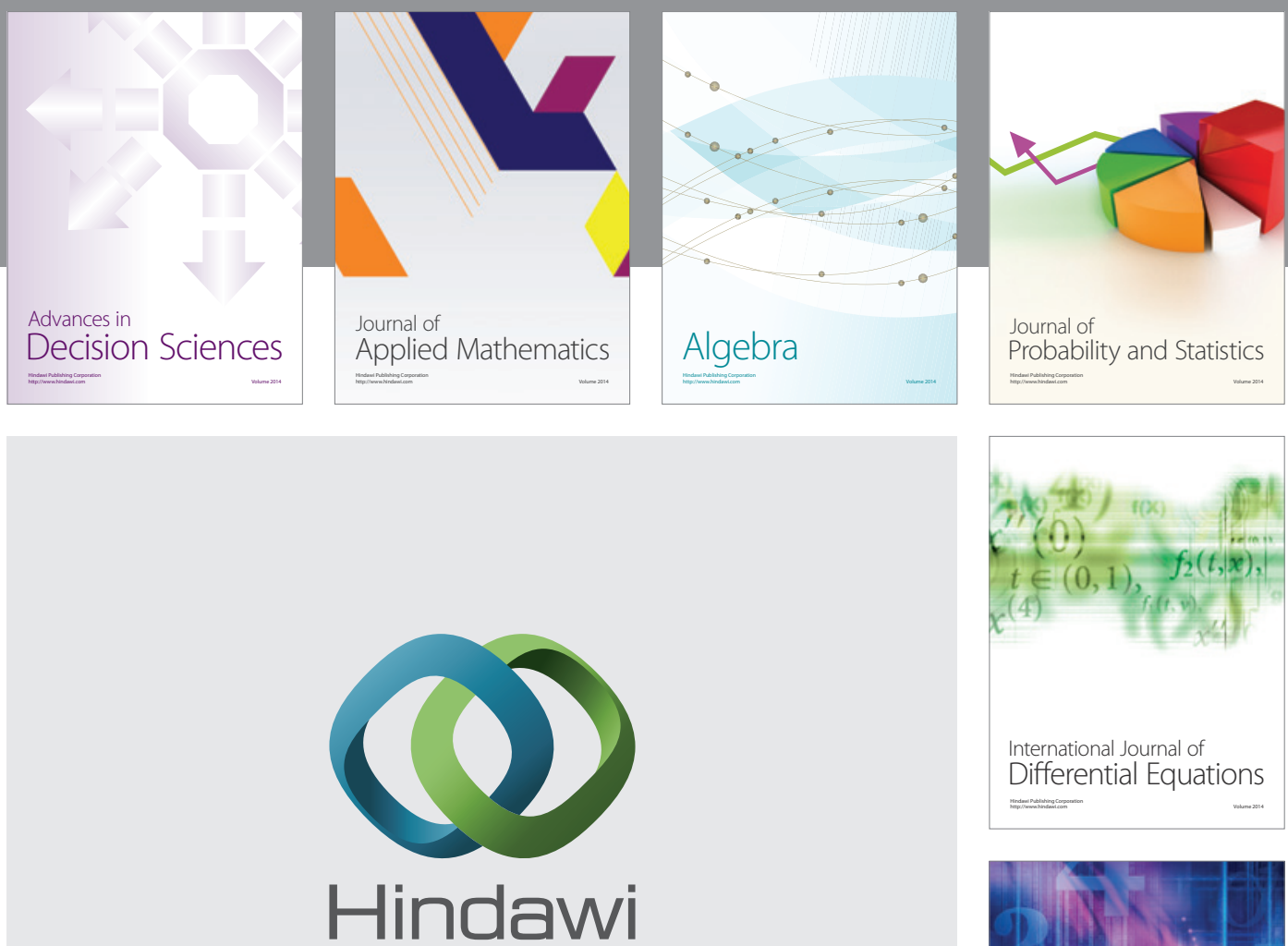

Submit your manuscripts at http://www.hindawi.com
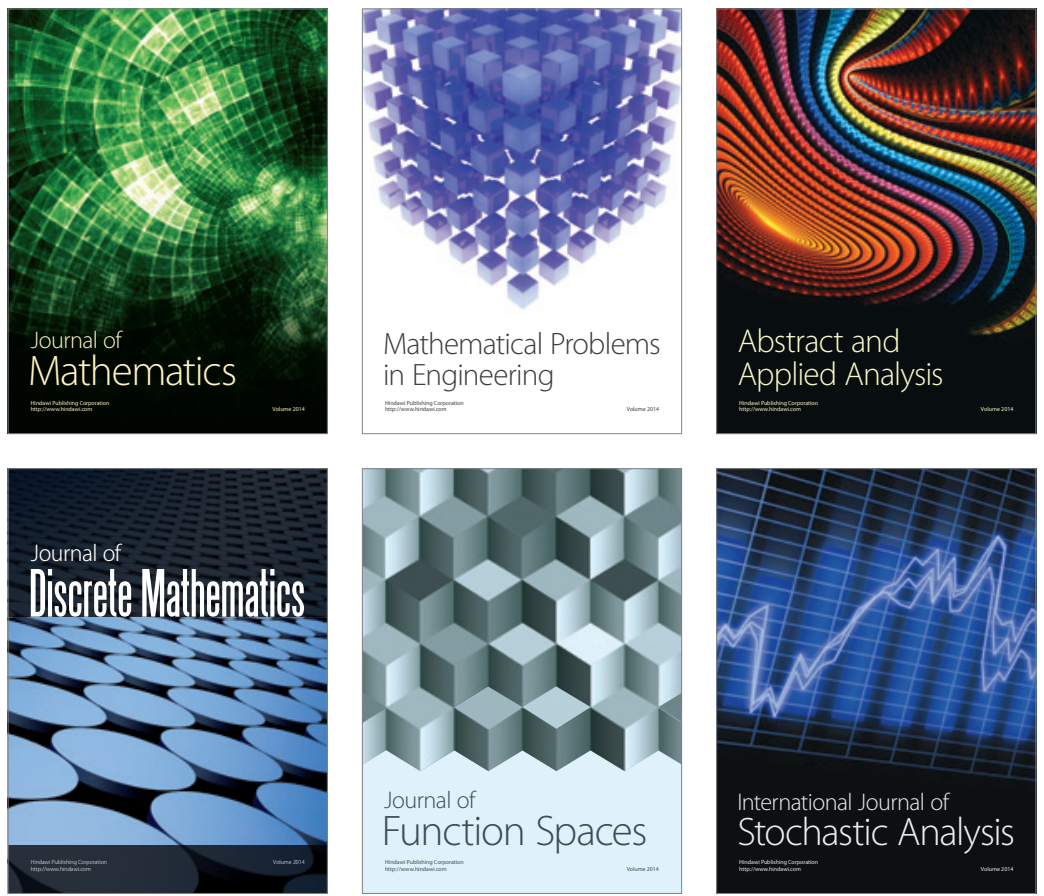

Journal of

Function Spaces

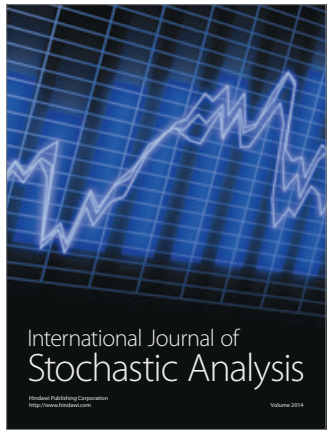

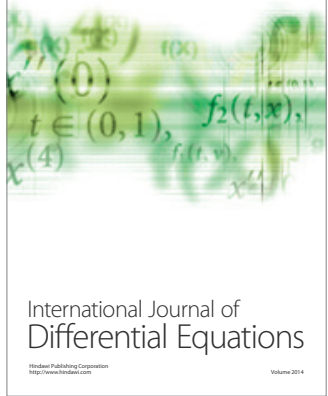
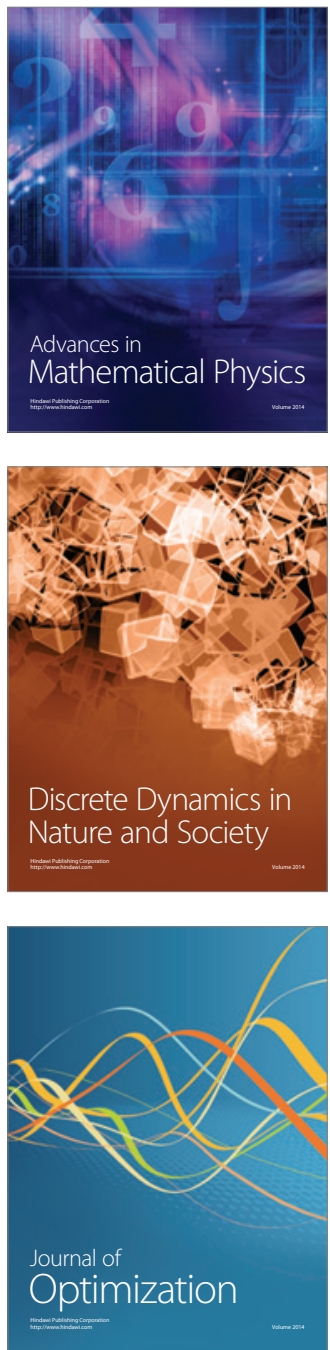